\title{
Improvement of corneal fluorescein staining in post cataract surgery of diabetic patients by an oral aldose reductase inhibitor, ONO-2235
}

\author{
H Fujishima, K Tsubota
}

Br J Ophthalmol 2002;86:860-863

\begin{abstract}
Aim: While the mechanism in the pathogenesis of diabetic corneal disease is unclear, aldose reductase has been implicated in corneal disease. The effects of an oral aldose reductase inhibitor (ARI) on the ocular surface of diabetic patients after cataract surgery were studied.

Methods: This clinical trial was designed to be randomised, double blinded, and placebo controlled. Pseudophakic patients with diabetes were randomly assigned to treatment with either oral ARI (ONO-2235) $(n=12)$ or placebo $(n=9)$ for 12 weeks. The vital staining of the ocular surface, tear production and clearance, break up time in tears (BUT), corneal and conjunctival sensation, and symptom score before treatments were examined as well as 4, 8, 12 weeks after the administration. Specular microscopic evaluation was also performed.

Results: After a 12 week period of oral ARI administration, fluorescein staining scores (from 2.04 (SD 1.12) to $1.46(1.18) ; p=0.016)$, conjunctival sensation (from 1.15 $(0.37)$ to $1.36(0.31) ; p=0.0006)$, and symptom scores (from 5.38 (1.932) to $4.00(2.07) ; p=0.0002)$ recovered significantly. Fluorescein staining of oral ARI administration also decreased compared with placebo $(p=0.017)$. Rose bengal staining, tear clearance, and corneal sensation were improved although this increase was minor. Tear production, BUT, and specular microscopic evaluation of the corneal epithelium and endothelium did not demonstrate a significant change.

Conclusion: Oral ARI opposes the ocular surface changes caused by diabetes, by recovery of ocular surface sensitivity as demonstrated through an improvement in vital staining.
\end{abstract}

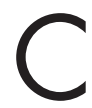
orneal epithelial abnormality in patients with diabetes mellitus has been a lively topic from the end of 1970s when ocular surgeries such as vitreous surgery gained popularity. Since that time, it is well understood that diabetes mellitus results in the ocular complications of cataract, retinopathy, and iritis. Patients with diabetes mellitus exhibit abnormalities of the corneal epithelium and endothelium, first observed in the 1980s using specular microscopy. Ocular surface disorders, such as corneal epithelites following ocular surgery, are thought to result from the disease progression of diabetes mellitus itself. ${ }^{1-3}$ Irrespective of ocular surgery, ${ }^{4-8}$ patients with diabetes experience a variety of corneal complications, including superficial punctate keratopathy, ${ }^{5}$ recurrent corneal erosion, ${ }^{9}$ persistent epithelial defect, or trophic ulceration. ${ }^{9}$

Although the molecular mechanism generating diabetic ocular surface abnormalities is unclear, the effects of aldose reductase, the first enzyme of the sorbitol pathway, in the complications in other tissues of diabetic patients suggests its involvement in the pathogenesis of this condition. ${ }^{10-12}$ The observation that diabetics demonstrate a decrease in corneal sensitivity ${ }^{6}$ and the complications of sterile ulcers in diabetic corneas suggests a neurotrophic involvement. ${ }^{9}$ Experimental studies described the effectiveness of aldose reductase inhibitors (ARI) in promoting corneal re-epithelialisation. ${ }^{13-15}$ The topical ARI, CT-112, was reported to improve corneal sensitivity, ${ }^{16}$ with some clinical benefits. The improvement of corneal sensation and tear dynamics in patients with diabetes mellitus also resulted from oral administration of ARI, ONO-2235 ((E)-3-carboxymethyl-5-[(E)-2-methyl-3-phenylpropenylidene] rhodanine). ${ }^{17}$ These data, although suggestive, are not conclusive. We therefore established a randomised, double blinded, and placebo controlled clinical trial, administering ARI orally for 12 weeks to patients with diabetes mellitus after cataract surgery to determine the effect of this treatment on the ocular surface condition of diabetic patients.

\section{MATERIALS AND METHODS \\ Patients}

A randomised, double blind, and placebo controlled study was performed to determine the efficacy of oral ARI in the treatment of diabetes mellitus epitheliopathy. This study was conducted in Ichikawa General Hospital, Tokyo Dental College, Chiba, Japan following approval by the institutional review board. Following their informed consent, we enrolled 27 patients with diabetes mellitus in this study who had undergone bilateral cataract extraction and implantation of posterior chamber intraocular lenses. There were no surgical complications, such as vitreous loss into the anterior chamber or haemorrhage. Diabetes mellitus was diagnosed by internal medicine doctors. Four patients discontinued their involvement in this study because of a lack of compliance or because of side effects of the medication and two patients did not perform all examinations; 21 patients (nine patients from the placebo controlled group) were followed for the duration of the study (Table 1). Although severe clinical abnormalities such as corneal ulcers or epithelial defect were not observed, all patients had had surgery performed from 3 to 9 months before the beginning of the study. All had symptoms such as foreign body sensation. ${ }^{18}{ }^{19}$ Four patients had pre-proliferative retinopathy, seven had proliferative retinopathy, 15 had neuropathy, and one had renal failure. Ten patients were treated with photocoagulation. Two of the placebo controlled patients had a history of vitrectomy surgery as a result of the vitreous haemorrhage.

\section{Drug administration}

After discussing the nature of the study with the patients, a total of $150 \mathrm{mg}$ of either placebo or ONO-2235 per day were administered three times daily for 12 weeks, 30 minutes before each meal. No other drug with known or suspected interactions with ONO-2235, or any systemic and topical 


\begin{tabular}{|c|c|c|}
\hline Patients & ARI & Placebo \\
\hline \multicolumn{3}{|l|}{ Sex } \\
\hline Men & 5 & 3 \\
\hline Women & 7 & 6 \\
\hline \multicolumn{3}{|l|}{ Type of DM } \\
\hline NIDDM & 11 & 8 \\
\hline IDDM & 1 & 1 \\
\hline \multicolumn{3}{|l|}{ Duration of DM } \\
\hline$<9$ years & 2 & 2 \\
\hline $10-19$ years & 5 & 5 \\
\hline $20-29$ years & 3 & 2 \\
\hline$>30$ years & 1 & 0 \\
\hline Unknown & 1 & 0 \\
\hline \multicolumn{3}{|l|}{ DM control } \\
\hline Food control & 5 & 2 \\
\hline Pill & 6 & 5 \\
\hline Insulin & 1 & 2 \\
\hline \multicolumn{3}{|l|}{ FBS } \\
\hline$<119 \mathrm{mg} / \mathrm{dl}$ & 2 & 0 \\
\hline $120-159 \mathrm{mg} / \mathrm{dl}$ & 5 & 4 \\
\hline$>160 \mathrm{mg} / \mathrm{dl}$ & 5 & 5 \\
\hline \multicolumn{3}{|l|}{$\mathrm{HbAlc}$} \\
\hline$<6.0 \%$ & 2 & 3 \\
\hline $6.1-9.0 \%$ & 9 & 5 \\
\hline$>9.0 \%$ & 1 & 1 \\
\hline \multicolumn{3}{|l|}{ Retina } \\
\hline Non-proliferative & 4 & 4 \\
\hline Pre-proliferative & 2 & 2 \\
\hline Proliferative & 4 & 3 \\
\hline Normal & 2 & 0 \\
\hline \multicolumn{3}{|l|}{ Photocoagulation } \\
\hline Done & 6 & 4 \\
\hline
\end{tabular}

drugs which have effects on tear production or the ocular surface, was prescribed during the study.

\section{Clinical examination}

Each measurement was made by the same investigator under the same conditions. Corneal sensation, rose bengal stain and fluorescein stain, tear break up time (BUT) ${ }^{20}$ Schirmer's test, and the cotton thread test, ${ }^{21}$ were used to evaluate the ocular surface. Central corneal sensation was measured with a Cochet and Bonnet aesthesiometer (Luneau Ophthalmologie, France). ${ }^{22}$ Rose bengal staining was graded from 0 to $3+$ at the nasal conjunctiva, the temporal conjunctiva, and the cornea (maximal grade of $9+$ ) ${ }^{23}$ Fluorescein staining was graded 0 to $9+$ at the cornea. ${ }^{23}$ BUT is defined as the number of seconds between the last complete blink and the first disturbance of the precorneal tear film. Schirmer's test was performed 5 minutes after instillation of a drop of solution containing $0.5 \%$ fluorescein and $0.4 \%$ oxybuprocaine hydrochloride solution into the cul de sac. ${ }^{18}$ Symptoms were recorded at the end of 4 , 8 , and 24 week treatment period by a single investigator under blinded conditions. Before treatment and 4, 8, and 12 weeks after treatment, subjective comfort was evaluated using a questionnaire giving a face score defined by nine faces, each showing a different expression. ${ }^{24}$ Specular microscopy was used to evaluate the corneal epithelium and endothelial changes.

\section{Statistical analysis}

Data were reported as real numbers. Statistical analysis utilised the paired $t$ test and Student's $t$ test. Probability values smaller than 0.05 are considered to be statistically significant.

\section{RESULTS}

Before treatment, mean corneal fluorescein staining score in these patients was 2.04 (SD 1.12). After a 12 week period of oral administration of ONO-2235, this value improved to 1.46 (1.18) $(\mathrm{p}=0.016)$. The score of the placebo group, however, did

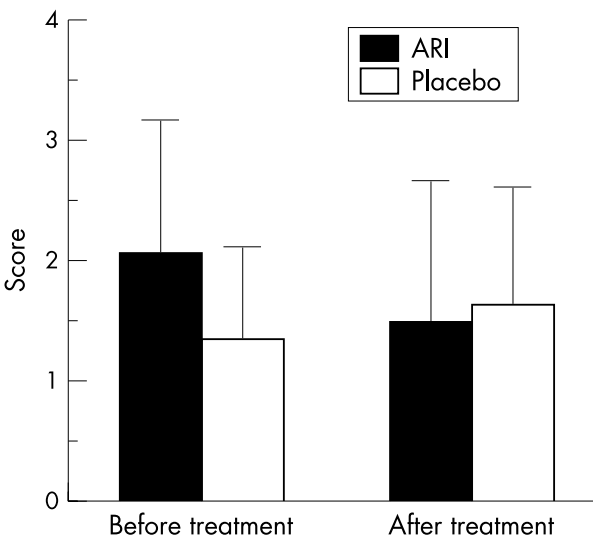

Figure 1 Corneal fluorescein staining. Vertical bar is SD; After treatment means after 12 weeks of treatment. After treatment of ONO-2235, fluorescein staining was significantly improved (from $2.04(1.12)$ to $1.46(1.18) ; p=0.016)$, but not of placebo (from 1.33 (0.77) to 1.61 (0.98); $p=0.31)$. Student's $t$ test demonstrated significant improvement within both groups $(p=0.017)$.

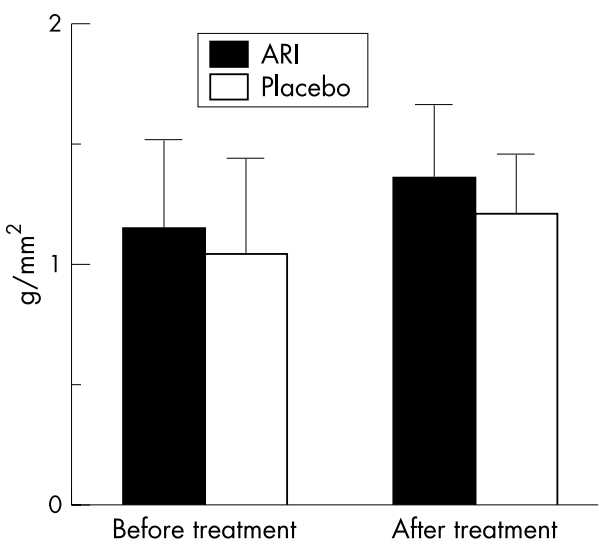

Figure 2 Conjunctival sensation. Vertical bar is SD; After treatmen means after 12 weeks of treatment. After treatment of ONO-2235 (from $1.15(0.37)$ to $1.35(0.31) ; p=0.0006$ ) and placebo (from $1.04(0.40)$ to $1.20(0.26) ; p=0.045)$, conjunctival sensation was significantly improved.

not improve significantly (from 1.33 (0.77) to 1.61 (0.98) $(p=0.31)$ (Fig 1$)$. Student's $t$ test demonstrated significant improvement within both groups $(\mathrm{p}=0.017)$. The recovery of fluorescein staining score paralleled the improvements observed in the conjunctival sensation (from $1.15(0.37)$ to 1.35 (0.31); ARI) ( $\mathrm{p}=0.0006)$ (Fig 2$)$ and the symptom score (from 5.25 (1.57) to 3.50 (2.06); ARI) $(p=0.0002)$ (Fig 3) following oral ONO-2235 administration. In the placebo treated patients, conjunctival sensation was also improved (from 1.04 $(0.40)$ to $1.20(0.26))(\mathrm{p}=0.045)$ although the symptom score did not improve (from 5.61 (1.38) to $4.89(1.32))(p=0.17)$. Student's $t$ test did not demonstrate a significant improvement within the two groups in either conjunctival sensation $(p=0.55)$ or symptom score $(p=0.40)$. Corneal fluorescein score improved in 4 weeks after administration, continuing to 8 and 12 weeks. Rose bengal staining (from 1.21 (1.02) to 0.83 (1.01); ARI), tear clearance (from 10.09 (13.90) to 9.45 (9.13); ARI), corneal sensation (from 4.58 (1.17) to 4.71 (1.24); ARI), tear production (from 9.56 (5.76) to 8.22 (5.58); ARI), BUT (from 3.64 (1.94) to 3.05 (1.29); ARI), and specular microscopic evaluation of the corneal epithelium (mean cell number from 58.50 (11.66) to 52.73 (17.49); ARI) and endothelium (mean cell number from 426.3 (190.1) to 447.9 (156.89); ARI) did not show significant changes. Although 


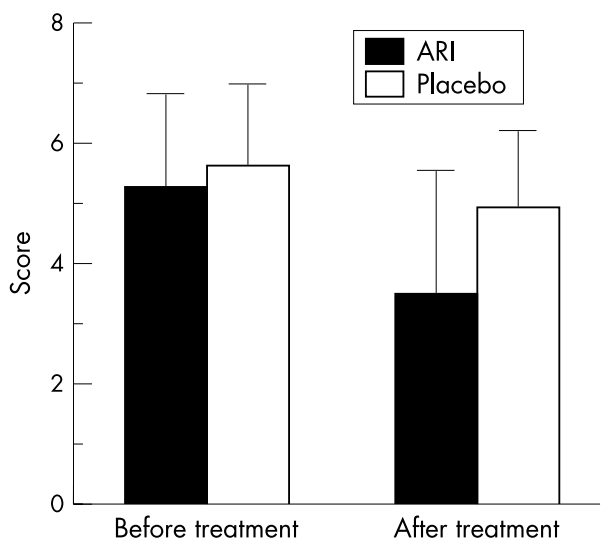

Figure 3 Symptom score. Vertical bar is SD; After treatment means after 12 weeks of treatment. After treatment of ONO-2235, symptoms score was significantly improved (from 5.25 (1.57) to 3.50 (2.06); $p=0.0002$ ), but not of placebo (from 5.61 (1.38) to $4.89(1.32) ; p=0.17)$.

four cases were dropped from the study because of urine colour changes or discontinuation of this drug, no additional side effects were seen in this treatment.

\section{DISCUSSION}

Diabetes causes a variety of clinical complications including diabetic corneal epitheliopathy. ${ }^{25}$ Various conditions contribute to retinal hypoxia; recently, however, the role of metabolic abnormalities associated with hyperglycaemia, such as the activation of the polyol pathway, has received worldwide attention. Corneal autofluorescence reported that the rate limiting enzyme in this metabolic pathway, aldose reductase, may be involved in the accumulation of pyridine nucleotides in the cornea. ${ }^{26}$ Theoretically, inhibition of this polyol metabolism enzyme reduces the impaired functions and histological changes in nerves. The onset and progression of diabetic complications may be prevented by ARI. ${ }^{1027-30}$

Oral ARI improves corneal epithelial changes caused by diabetes, through the recovery of corneal sensation and increased tear production. ${ }^{17}$ The study, an open label trial, allowed both the patients and examiners to be aware when active medication was being given, possibly biasing the results. To explore the clinical safety and benefits of oral ARI, we conducted a randomised and controlled clinical study of oral ARI. Corneal fluorescein staining was significantly improved compared with the placebo, with parallel improvements in conjunctival sensation and symptom score. The distribution of fluorescein scores also revealed that women and older patients had increased staining over placebo groups (data not shown). Improvement in the dynamics of tear production may result effective suppression of aldose reductase activity in the Schwann cells in a similar way to "dry eye" patients. ${ }^{31}$ Improvement of tear dynamics may improve the conjunctival and corneal abnormalities seen in "dry eye." Improvements in rose bengal and fluorescein staining scores concurrent with a reduction of the debris in tears is attributable to improved tear dynamics.

Before treatment, the corneal fluorescein staining score of the ARI treating group was higher than the placebo group. We have no idea why it happened, but we thought it happened by chance because patients were enrolled after the double blind study.

Topical oxybuprocaine (proparacaine) increases tear film osmolarity in rabbit eyes, suggesting that the increase results from decreases in tear secretion secondary to decreased ocular surface sensitivity. ${ }^{32}$ In this study, although cornea and conjunctival sensation demonstrated an increasing trend, significant increase were observed only for conjunctiva. The improvement of symptom score may also be due to these slight improvements of tear production dynamics and conjunctival sensitivity resulting from drug treatment. All these significant improvements may be due to this drug.

Although previously surgery was an extensive procedure, ${ }^{133}$ recent advances in cataract operation procedures resulting in reduced inflammation and operation time has led to decreased side effects. In this study, the ocular surface demonstrated less optical damage (our former report included two patients with corneal epithelial defect and two with corneal ulcer) ${ }^{17}$; and were better controlled, although only fluorescein staining and not other tear factors improved. Rose bengal staining, tear clearance, and corneal sensation were improved, but tear production or BUT, and specular microscopic evaluation for corneal epithelium and endothelium did not improve significantly. These factors may result from improvements in surgical techniques. In addition, the two case of vitrectomy belonged in the placebo control group, possibly affecting the results. These results suggest that fluorescein staining is the most sensitive measurement; this method should be used to evaluate ocular surface conditions in patients with diabetes mellitus.

In the present study, after treatment with oral ONO-2235, at $150 \mathrm{mg} /$ day for 12 weeks, improved the abnormalities associated with diabetes, including ocular surface disorders and symptoms. In an animal model, the serum density of oral ONO-2235 amounted to a maximum density of 15 minutes after oral administration in male rats. ${ }^{34}$ Following a single oral administration, choroidal density peaked 1-3 hours with a serum density approximately $20 \%$ that of male rat. We have not clarified how ARI reaches the cornea. However it has been reported that positive radioactive findings were observed by radioimmunological assay in ocular tissues, Harder's gland (rat specific accessory lacrimal gland) and the lens in rats after oral administration of ${ }^{14} \mathrm{C}$ labelled ARI. ARI probably reaches the cornea via tears or aqueous humour. ${ }^{34}$ Four patients discontinued their involvement in the study because of colour change in urine or other problems, not because of systemic side effects of this drug. ONO-2235, $150 \mathrm{mg} /$ day, is safe, making it a promising candidate for effective treatment in human eyes. Administration of ONO-2235 for 12 weeks to patients with diabetes and a diabetic ocular surface condition led to improvements in tear dynamics and the symptoms of postcataract extraction keratopathy.

\section{ACKNOWLEDGEMENTS}

We thank Ms Yukiko Yagi and Ms Saori Nishijima for their expert clinical examinations of ocular surfaces.

\section{Authors' affiliations}

H Fujishima, K Tsubota, Department of Ophthalmology, Tokyo Dental College and Department of Ophthalmology, Keio University School of Medicine, Japan

Correspondence to: Hiroshi Fujishima, MD, Department of Ophthalmology, Tokyo Dental College, 5-1 1-13 Sugano, Ichikawa, Chiba, Japan 272-8513; fujishim@tdc.ac.jp

Accepted for publication 14 March 2002

\section{REFERENCES}

1 Brightbill FS, Myers FL, Bresnick GH. Postvitrectomy keratopathy. Am J Ophthalmol 1987;85:651-5.

2 Perry HD, Foulks GN, Thoft RA, et al. Corneal complications after closed vitrectomy through the pars plana. Arch Ophthalmol 1978;96:1401-3.

3 Michels RG, Ryan SJ. Results and complications of 100 consecutive cases of pars plana vitrectomy. Am J Ophthalmol 1975;80:24-9.

4 Schultz RO, Matsuda M, Yee RW, et al. Corneal endothelial changes in type I and type II diabetes mellitus. Am J Ophthalmol 1984;98:401-10.

5 Schultz RO, Horn DLV, Peters MA, et al. Diabetic keratopathy. Trans Am Ophthalmol Soc 1981;79:180-99.

6 Schwartz DE. Corneal sensitivity in diabetics. Arch Ophthalmol $1974 ; 91: 174-8$ 
7 Snip RC, Thoft RA, Tolentino FI. Similar epithelial healing rates of the corneas of diabetic and nondiabetic patients. Am J Ophthalmol 1980;90:463-8

8 Tsubota K, Chiba K, Shimazaki J. Corneal epithelium in diabetic patients. Cornea 1991;10:156-60.

9 Hyndiuk RA, Kazarian EL, Schultz RO, et al. Neurotrophic corneal ulcers in diabetes mellitus. Arch Ophthalmol 1977;95:2193-6.

10 Kinoshita JH, Fukushi S, Kador P, et al. Aldose reductase in diabetic complications of the eye. Metabolism 1979;28:462-9.

11 Cogan DG, Kinoshita JH, Kador PF, et al. Aldose reductase and complications of diabetes. Ann Intern Med 1984;101:82-91.

12 Terashima $\mathbf{H}$, Hara K, Yamamoto R, et al. Effects of a new aldose reductase inhibitor on various tissues in vitro. J Pharmacol Exp Ther 1984;229:226-30.

13 Kern TS, Engerman RL. Distribution of aldose reductase in ocular tissues. Exp Eye Res 1981;33:175-82.

14 Fukushi S, Merola O, Tanaka M, et al. Reepithelialization of denuded corneas in diabetic rats. Exp Eye Res 1980;31:61 1-21.

15 Datiles MB, Kador PF, Fukui HN, et al. Corneal re-epithelialization in galactosemic rats. Invest Ophthalmol Vis Sci 1983;24:563-9.

16 Hosotani H, Ohashi Y, Yamada M, et al. Reversal of abnorma epithelial cell morphology and reduced corneal sensitivity in diabetic patients by aldose reductase inhibitor, CT-112. Am J Ophthalmol 1995; 119:288-94.

17 Fujishima H, Shimazaki J, Yagi Y, et al. Improvement of corneal sensation and tear dynamics in diabetic patients by oral aldose reductase inhibitor, ONO-2235: a preliminary study. Cornea 1996;15:368-72.

18 Tsubota K, Toda I, Yagi Y, et al. Three different types of dry eye syndrome. Cornea 1994:13:202-9.

19 Ogawa Y, Kamoshita I, Yoshino K, et al. Dry eye in diabetes mellitus. Atarashii Ganka 1992;9:1867-70.

20 Paschides CA, Kitsios G. Evaluation of tear break-up time, Schirmer's-1 test and rose bengal staining as confirmatory tests for keratoconjunctivitis sicca. Clin Exp Rheumatol 1989;7:155-7.

21 Sakamoto R, Bennet ES, Henry VA, et al. The phenol red thread tear test: A cross-cultural study. Invest Ophthalmol Vis Sci 1993;34:3510-4.

22 Cochet P, Bonnet R. L'esthesiometrie corneenne: realisation et interet pratique. Bull Soc Ophtalmol Fr 1961;61:541-50
23 Toda I, Tsubota K. Practical double-vital staining for ocular surface evaluation. Cornea 1993;12:366-8.

24 Tsubota K, Goto E, Fujita $\mathrm{H}$, et al. Treatment of dry eye by autologous serum application in Siogren's syndrome. Br J Ophthalmol 1999:83:390-5

25 Hotta N, Kakuta H, Ando F, et al. Current progress in clinical trials of aldose reductase inhibitors in Japan. Exp Eye Res 1990;50:625-8.

26 Ishino Y, Yokoi N, Yasuhara T, et al. Investigation of corneal autofluorescence in diabetic patients. J Jpn Ophthalmol Soc 2000; 104:572-6.

27 Hotta N, Kakuta H, Ando F, et al. Clinical trial of aldose reductase inhibitor ONO-2235: effect on diabetic retinopathy. Amsterdam: Excerpta Medica 1988:253-66. (Sakamoto N, Kinoshita JH, Kador PF, Hotta N, eds. Polyol pathway and its role in diabetic complications)

28 Hotta N, Kakuta H, Koh N, et al. In vitro retinal and erythrocyte polyol pathway regulation by hormones and an aldose reductase inhibitor. Diabetes Res Clin Pract 1991;14:29-36.

29 Hotta N, Kakuta H, Fukasawa $\mathrm{H}$, et al. Effects of a fructose-rich diet and the aldose reductase inhibitor, ONO-2235, on the development of diabetic neuropathy in streptozotocin-treated rats. Diabetologia 1985;28:176-80.

30 Matsuda $M$, Awata T, Ohashi $Y$, et al. The effects of aldose reductase inhibitor on corneal endothelial morphology in diabetic rats. Curr Eye Res 1987;6:391-7

31 Gabbay KH, O'Sullivan JB. The sorbitol pathway: enzyme localization and content in normal and diabetic nerve and cord. Diabetes 1968;17:239-43.

32 Gilbard JP, Gray KL, Rossi SR. A proposed mechanism for increased tear-film osmolarity in contact lens wearers. Am J Ophthalmol 1986:102:505-7.

33 Foulks GN, Thoft RA, Perry HD, et al. Factors related to corneal epithelial complications after closed vitrectomy in diabetics. Arch Ophthalmol 1979:97:1076-8.

34 Miyamoto S, Kajiwara I, Sawada M, et al. The distribution of ONO-2235 density in a living body after oral administration in rat. Gendai iryo 1986;51:51-81.

\section{Rapid responses}

Letters on the following British Journal of Ophthalmology papers have been published recently as rapid responses on the BJO website. To read these letters visit www.bjophthalmol.com and click on "Read eletters"

Value of two mortality assessment techniques for organ cultured corneal endothelium: trypan blue versus TUNEL technique. P Gain, $G$ Thuret, $C$ Chiquet, et al. Br J Ophthalmol 2002;86:306-10.

Use of bovine pericardium as a wrapping material for hydroxyapatite orbital implants. M Gupta, P Puri, I G Rennie. Br J Ophthalmol 2002;86:288-9.

If you would like to post an electronic response to these or any other articles published in the journal, please go to the website, access the article in which you are interested, and click on "eletters: Submit a response to this article" in the box in the top right hand corner. 